\title{
8. Un cas de proverbalisation en diachronie
}

Olof Eriksson

Linné universitetet

\section{Introduction}

Cette étude porte sur les aspects diachroniques du phénomène linguistique que nous proposons d'appeler proverbalisation (Eriksson 2008 ; 20I0), que nous avons, dans des études antérieures, appelé suppléance verbale (Eriksson I985; 2006 ; cf. : Moignet I960: suppléance du verbe) et qu'on nomme généralement en linguistique anglo-saxonne verbal substitution (voir en particulier Halliday et Hasan I976). On a aussi emprunté le terme anglais pour l'appliquer au français : substitution verbale (Apothéloz I995).

C'est le linguiste danois Louis Hjelmslev qui, dans un article publié en I937, a lancé le terme de proverbe (1937 : 57) (ou de pronom verbal [ibid.] ; cf. pour le suédois Teleman et al. I999a : 214 et I999b : pronominell verbfras et Thorell $\mathrm{I} 977: 76:$ pronominellt verb) et qui en a souligné le caractère indispensable, au même titre - et au même degré que le pronom, dans toute langue possédant la catégorie du verbe. Or, avant lui, en I 904 déjà, le linguiste suédois Adolf Noreen avait parlé de proverba dans le tome $\mathrm{V}$ de sa monumentale grammaire de la langue suédoise (I904-I9I $2: 67$ ).

Nous ne nous occuperons pas ici des aspects théoriques de la proverbalisation. Nous l'avons fait de façon approfondie dans notre monographie de I985. Disons seulement qu'elle est syntaxiquement plutôt que stylistiquement motivée, comme le montre déjà l'exemple (I). Il ne s'agit pas, comme le disent les Le Bidois dans le deuxième tome

Comment citer ce chapitre :

Eriksson, Olof, Un cas de proverbalisation en diachronie. In: Engwall, Gunnel \& Fant, Lars (eds.) Festival Romanistica. Contribuciones lingüisticas - Contributions linguistiques - Contributi linguistici - Contribuições linguísticas. Stockholm Studies in Romance Languages. Stockholm: Stockholm University Press. 201 5, pp. I60-I74. DOI: http://dx.doi.org/IO.I6993/bac.h. License: CC-BY 
de leur syntaxe, et comme le disent d'ailleurs la plupart des linguistes qui se sont exprimés en la matière, d'un procédé " qui s'explique par le désir de varier l'expression »(Le Bidois \& Le Bidois I97I : 289), mais d'un instrument de représentation syntaxique dans le domaine verbal, comme c'est le cas de la pronominalisation dans le domaine nominal. On constate, dans (Ia), que le verbe répété (frapper) n'aurait pas la «faculté représentative » qui permet au verbe faire de se charger, dans la comparative, de la complémentation du verbe principal par trois adverbiaux exprimant successivement l'itération de l'action (deux ou trois fois), sa localisation (sur la table) et l'instrument avec lequel elle s'exécute (avec son dé); c'est un élément de simple reprise, non de représentation, d'où l'agrammaticalité de (Ib) et de (Ic) :

(гa) Elle frappa deux ou trois fois sur la table avec son dé, comme font souvent les couturières (Georges Duhamel, Confession de minuit, Mercure de France, r948: 97)

(rb) *Elle frappa deux ou trois fois sur la table avec son dé, comme frappent souvent deux ou trois fois sur la table avec leur dé les couturières.'

(Ic) *Elle frappa deux ou trois fois sur la table avec son dé, comme frappent souvent les couturières.'

Ce que nous nous proposons modestement de faire ici, c'est un survol de l'évolution en français d'un cas particulièrement intéressant de proverbalisation, à savoir la construction qu'on voit schématisée et exemplifiée dans (2) :

(2a) $\mathrm{X}-\mathrm{V}_{\mathrm{I}}-\mathrm{O}_{\mathrm{I}}-$ Connecteur comparatif (CC) $-\mathrm{X} / \mathrm{Y}-\mathrm{V}_{\mathrm{I}}-\mathrm{O}_{2}$

(2b) Ils $(\mathrm{X})$ le $\left(\mathrm{O}_{\mathrm{I}}\right)$ traitaient $\left(\mathrm{V}_{\mathrm{I}}\right)$ comme $(\mathrm{CC})$ on $(\mathrm{X} / \mathrm{Y})$ traite $\left(\mathrm{V}_{\mathrm{I}}\right)$ un chien $\left(\mathrm{O}_{2}\right)$

Il s'agit donc du cas où, dans une comparative, le verbe est identique à celui de la principale tout en ayant un objet différent de celui de la principale, cas qui présente une concurrence, dans la comparative, entre les trois procédés d'implication ('Ils le traitaient comme un chien'), de répétition ('Ils le traitaient comme on traite un chien') et de proverbalisation ('Ils le traitaient comme on /le/ fait d'un chien'). Pour une analyse des mécanismes de cette concurrence, nous renvoyons à Eriksson I985: 76-I26. Pour une analyse de la proverbalisation en français médiéval, et avec des exemples de la construction " objective » tirés de l'ensemble de cette période, on se reportera en premier lieu à Ponchon I994 : 25 I-34 I, mais aussi à Damourette et Pichon I936: I28-I33 et à Moignet I960: I3-24, I07-I24. 
Nous n'entrerons pas ici dans les détails de la discussion des aspects théoriques de la construction " objective ", discussion qui concerne surtout la question des raisons de l'introduction d'une préposition (de, pour, avec, à) entre proverbe et objet, celle de la généralisation subséquente de l'usage de la construction "prépositionnelle » et celle de la tendance actuelle à l'emploi exclusif, dans ce rôle, de la préposition avec, résultat, à notre avis, d'un processus de grammaticalisation de cette préposition ( $c f$. Eriksson 2008).

\section{Analyse diachronique}

La première attestation du verbe faire en français se trouve dans les Serments de Strasbourg déjà et c'est justement en tant que proverbe encore que dans une conditionnelle et non pas dans une comparative qu'il y apparaît ( Ia). La même phrase illustre la concurrence que se font depuis toujours, en comparative, les procédés de répétition (salvar) et de proverbalisation. La traduction en français moderne donnée en ( $\mathrm{I} b$ ) est presque identique à celle donnée par Ferdinand Brunot (I966 : I44) :

(ra) [...] si salvarei eo cist meon fradre Karlo, et in aiudha et in cadhuna cosa, si cum om per dreit son fradra salvar dift, in o quid mi altresi fazet,...

( $\mathrm{rb}$ ' 'je soutiendrai mon frère Charles de mon aide et en toute chose, comme on doit justement le faire pour/soutenir son frère, à condition qu'il me soutienne à son tour / m'en fasse autant'

Dès les plus anciens textes et jusqu'à la fin du Moyen Âge, c'est la construction " directe ", sans intermédiaire prépositionnel, qui règne de façon absolue :

(2) Plus aimet il traïsun et murdrie qu'il ne fesist trestut l'or de Galice (La Chanson de Roland; éd. Moignet, Bordas, I969, v. I476)

Ainsi, par exemple, dans l'ensemble des cinq livres de Chrétien de Troyes, on compte au total I7 exemples de la construction " objective ", tous avec objet construit directement (3-6). Les exemples (5-6) font voir à quel degré, du temps de Chrétien, le statut du verbe faire en tant que proverbe était encore incertain, en (5) par l'inclusion dans la représentation de faire à la fois d'un objet direct et d'un objet indirect ('le sert et lui témoigne beaucoup d'honneur'), en (6) par l'antéposition de l'objet (la soë) et l'accord au féminin du proverbe avec cet objet (faite), effet, dans les deux cas, d'une confusion entre faire verbe 
« plein » et l'auxiliaire qu'est en fonction proverbale ce verbe ('pas plus qu'elle n'avait fait de la sienne') :

(3) Fenice en mene, si s'en vont.

$\mathrm{Ne}$ finent tant qu'en Grece sont

$\mathrm{O}$ a grant joie le recevent

Si com lor seignor faire devent (Chrétien, Cligès, éd. Méla et Collet, Le Livre de Poche, I994, v. 6665-6668)

(4) Jo te dirai : ce est ma lance.

Dites vos, fait il, qu'an la lance

Si con je faz mes javeloz ? (Id., Perceval, éd. Méla, Le Livre de Poche, I990, V. I9I-I93)

(5) Et neïs la fille au seignor

Le sert et porte grant honor,

Comme on doit faire son boin hoste (Id., Yvain, éd. Hult, Le Livre de Poche, I993, v. 5407-5409)

(6) Et il se couche tot a tret, Mes sa chemise pas ne tret

Ne plus qu'ele ot la soë faite (Id., Lancelot, éd. Méla, Le Livre de Poche, I992, v. I2I3-I2I5)

En ancien français, la constitution de l'objet, contrairement au français moderne, n'affecte pas le déclenchement de la proverbalisation. C'est ainsi qu'on trouve fréquemment l'objet direct en forme du cas oblique du pronom personnel (7-9), emploi impossible en français moderne ( $c f$. l'anglais moderne : "You don't love me as much as I do you »; cf. [9]) :

(7) Or m'est il solaz et deliz

De tes mançonges escouter,

Q'ansin orroie je conter

Un fableior com je fais toi (Chrétien, Perceval, éd. Méla, Le Livre de Poche, I990, v. 8588-859I)

(8) Mais ne regardoit mie mains

La damoisele le vassal

De bon huil et de cuer leal

Qu'il fesoit li par contençon (Id., Erec et Enide, éd. Fritz, Le Livre de Poche, I992, I494-I497)

(9) $-[\ldots]$ mais je vos aim plus que vos ne faciés mi.

- Avoi ! Fait Aucassins, bele douce amie, ce ne porroit estre que vos m'amissiés tant que je faz vos (Aucassain et Nicolete, éd. Roques, I936: XIV, v. I6-I 8 ) 
Cette syntaxe est encore bien vivante aux $\mathrm{XV}^{\mathrm{e}}$ et $\mathrm{XVI} \mathrm{I}^{\mathrm{e}}$ siècles, en principale (IO) aussi bien qu'en comparative (II) et elle s'étend également à l'objet indirect (I2) :

(Io) Il me salua ; si feis je luy (Les Cent Nouvelles Nouvelles, éd. Jourda, Gallimard [Pléiade], I956:32)

(I I) Je ne veulx poinct aussi nyer que, estant en ung lieu si privé et hors de tout soupson, je ne l'aye baisé de meilleur cueur que je ne faictz vous (Marguerite de Navarre, L'Heptaméron, Gallimard [Pléaide], I956:8 18 )

(I2) En mi la cort au vavasor,

Cui Dex doint et joie et enor

Tant com il fist moi cele nuit,

Pendoit une table ;... (Chrétien, Yvain, éd. Roques, I960, v. 209-2 I 2)

Au XVII siècle, certainement sous l'impulsion du débat que soulevaient à l'époque classique les questions de norme en matière de syntaxe, il y eut une réaction contre cet usage. Ce débat fit naître le sentiment qu'il était plus logique de placer le pronom, en fonction d'objet direct ( $13-\mathrm{I} 4$ ) ou indirect ( $\mathrm{I} 5-\mathrm{I} 6$ ), sous sa forme atone devant faire. Tout logique qu'il était, cet usage ne semble pas avoir survécu au XVIII ${ }^{\mathrm{e}}$ siècle; on ne le trouve plus après I 800 :

(I3) Et puisque par ce choix Albe montre en effet Qu'elle m'estime autant que Rome vous a fait (Corneille; cit. Moignet I960: II3)

(I4) Il faut que j'éveille les autres et que je les tourmente comme on $m$ 'a fait (Molière ; cit. Livet I 896:320)

(I 5) Il ne vous auroit pas joué le tour qu'il vous a fait (ibid.)

(I6) [...] mais ne vous avisez pas de lui serrer la main comme je vous fais, et de l'embrasser comme je vous embrasse (Louvet, Les Amours du Chevalier de Faublas [1787], Gallimard [Pléiade], I966-I969: t. II : 432)

Face à l'impossibilité de l'une et de l'autre de ces structures en français moderne, celui-ci se débrouille en faisant appel à l'intermédiaire d'une préposition entre proverbe et objet. Avec l'introduction de la préposition, il est redevenu possible, comme on l'avait fait jusqu'au XVI siècle, de faire suivre le proverbe de la forme tonique du pronom personnel, qui se voit assigner ainsi le rôle de régime de la préposition : 
(I7) Si je m'autorise à les tromper, quel que soit le prétexte, je les encourage à la pareille : à me traiter comme j'ai fait d'eux (Vercors, Les yeux et la lumière, Minuit, I948: 82)

(I8) Quand elle aura eu son enfant tu t'occuperas de lui, tu l'élèveras comme tu as fait pour moi (Paul Vialar, La Grande Meute, Fayard, I95 I : 93)

En fonction d'objet indirect, la préposition est pour (I9) ou avec (20), à l'exclusion de à, employée, elle, devant un objet substantival (2I), en concurrence toutefois avec pour (22) et avec (23):

(19) Je criais à mon tour, comme l'avait fait Sarah pour moi : "Attention, veux-tu... » (Max Gallo, Crépuscule, Le Livre de Poche, I98I : 287)

(20) J'ai envie de lui dire comme Thérèse le fait avec moi : "Essuie-toi, va te laver les dents " (Id., L'Oisean des origines, Robert Laffont, I974: 25)

(2I) J'hésitais aussi à le [le sucre] leur présenter dans ma main ouverte, comme je l'avais lu que l'on fait aux chevaux (Jacques Borel, L'Adoration, Gallimard, I965: I08)

(22) Elle répétait à Ann les noms comme on le fait pour les enfants auxquels on veut donner des repères (Max Gallo, France, Grasset, I980: I32)

(23) Et, comme autrefois, quand vous parler m'intimidait trop, je vous écris. Comme je faisais aussi avec mon mari, dans les premiers temps (Henry de Montherlant, Les Lépreuses, Gallimard [Pléiade], I959 : I 53I)

Ce qui vaut pour ce cas spécial de la construction " objective " vaut aussi pour son développement général en français : de directe, la construction est devenue indirecte, prépositionnelle (pour les causes de ce développement, voir Moignet 1960 ; Pinchon I972 ; Eriksson I985). On a relevé de cette dernière des exemples isolés antérieurs à I 500 (cf. Moignet I960 et Ponchon I994). Or, si par genèse de la construction indirecte on entend une quelconque systématisation de son usage, il faut la dater de la première moitié du XVI siècle, où elle apparaît de façon récurrente dans l'œuvre de Rabelais. L'ensemble de ses cinq livres donne pour la construction « objective »2 I exemples, dont 7 avec objet construit indirectement (tous avec la préposition de) (24-26). L'exemple (26) est particulièrement intéressant parce qu'il 
montre l'attitude vacillante de Rabelais à l'égard de l'emploi de la préposition; il y change de construction d'une phrase à l'autre :

(24) En icelle facon, saulva, après Dieu, ladicte Arche de periller, car il lui bailloit le bransle avecques les jambes, et du pied la tournait où il vouloit, comme on faict $d u$ gouvernail d'une navire (Rabelais, Pantagruel [1 532], Garnier, I965, I : 227)

(25) Par Golfarin, nepveu de Mahon, si tu bouges d'ici, je te mettray au fond de mes chausses, comme on faict d'un suppositoire (Ibid. : 363 )

(26) [...] mais ilz ne sceurent si bien faire que le jarret ne luy en demourast comme il le tenoit, et le mangeoit trés bien, comme vous feriez $d$ 'une saulcisse ; et quand on luy voulut oster l'os, il l'avalla comme un cormoran feroit un petit poisson (Ibid.: 236)

En dehors de l'œuvre de Rabelais, on ne trouve, au XVI ${ }^{\mathrm{e}}$ siècle, que des exemples isolés de la nouvelle syntaxe (27-28). Par exemple, des Io exemples de la construction " objective » relevés dans L'Heptaméron de Marguerite de Navarre, 2 seulement présentent une préposition (de) devant l'objet (27):

(27) Il me semble que c'est beaucoup mieulx faict d'aymer une femme comme femme, que d'en ydolatrer plusieurs comme on fait d'une ymaige (M. de Navarre, L’Heptaméron [I 558 ], Gallimard [Pléiade], 1956: 793)

(28) Aux bains, que les anciens prenoyent tous les jours avant le repas, et les prenoyent aussi ordinairement que nous faisons de l'eau à laver les mains, ils ne se lavoyent du commencement que les bras et les jambes (Montaigne, Essais, Gallimard [Pléiade], I976 : 28 5-286)

En français classique, la construction directe domine toujours fortement. Les exemples du type illustré par (29-34) abondent chez les grands auteurs du XVII siècle (cf. Fournier \& Fuchs I999, pour l'usage de la proverbalisation en français classique) :

(29) Vous devriez l'apprendre, Monsieur, comme vous faites la danse (Molière ; cit. Livet I896, II : 320)

(30) Je veux bien que vous me traitiez comme on fait les dieux (La Fontaine; cit. Regnier I 892, I : 367)

(3I) Je te traiterois comme j'ai fait mon frère (Corneille ; cit. MartyLaveaux I868: 4I9) 
(32) Dieu tolère le socinianisme, comme il fait les autres sectes (Bossuet; cit. Quillacq I903:4II)

(33) $[\ldots]$ on examina mon amusement comme on aurait fait une tragédie (Racine ; cit. ibid. : 4I2)

(34) $[\ldots]$ on regarde une femme savante, comme on fait une belle arme (La Bruyère ; cit. ibid. : 4I 2 )

C'est seulement le dépouillement de textes rédigés dans un style moins soutenu, moins " littéraire », qui permet de relever, pour la période du français classique, des exemples de la nouvelle syntaxe. Par exemple, dans une lettre écrite en I697 par Mme de Maintenon et adressée à l'Archevêque de Paris, elle s'exprime en ces termes :

(35) Et, quand vous censurerés le livre [celui de Fénelon], ne le regardera-t-il [le duc de Beauvillier] pas comme il a fait de ceux de Mme de Guyon, dont il se desfit, dès que son Archevesque les eust deffendus (Maintenon, Lettres, IV : I 50, éd. Marcel Langlois, Paris, Letouzey \& Ané, I935-1939)

Et dans la correspondance entre Mme de Maintenon et la Princesse Des Ursins, on trouve, sous la plume plus spontanée de cette dernière, plusieurs exemples dans le genre de ceux-ci :

(36) Ceci doit vous persuader, madame, qu'au lieu d'oublier ma nation, je ressens vivement qu'on la veut avilir ; je l'aime comme une bonne mère fait de sa fille, qui ne la flatte pas dans ses défauts, et qui se complaît dans ses bonnes qualités (Des Ursins, Lettres, IV : 370 [année I709], Paris, Bossange Frères, I 826)

(37) Si le cardinal des Gindice parlait aussi bien français qu'il fait de sa langue naturelle, son esprit en brillerait encore davantage (Ibid. : 438 [année I7I4])

Or, pour voir l'usage de la construction indirecte se généraliser tant soit peu, il faut attendre le milieu du XIX ${ }^{\mathrm{e}}$ siècle, où des auteurs aussi différents que Nerval (38), Dumas père (39) et Sainte-Beuve (40) s'en servent régulièrement :

(38) Il emplit de paille un grand sac qu'il sangla sur son cheval, et prit dans ses bras l'abbesse comme il eût fait d'un enfant (Nerval, Le Marquis de Fayolle, Gallimard [Pléiade], r960: 7 1 8)

(39) Le brigadier se fit donc apporter un fagot et de la paille ; il bourra la cheminée comme il eût fait d'un mortier, et y mit le feu (Dumas, Le Comte de Monte-Cristo, Gallimard [Pléiade], I98 I : I I94) 
(40) On conçoit que [...] ils en sachent un gré infini à leurs intrépides devanciers, et environnent leurs noms d'une sorte de consécration scientifique, comme les religions naissantes ont fait pour leurs précurseurs et leurs martyrs (Sainte-Beuve, Premiers lundis, Gallimard [Pléiade], I966: 277)

Et vers la fin du siècle, un auteur comme Guy de Maupassant ne semble pas connaître d'autre construction :

(4I) Une forte paysanne le saisit dans ses bras et l'emporta comme elle eût fait d'un petit enfant (Maupassant, Une vie, Le Livre de Poche, I966: 209-2 I0)

(42) [...] et à la sortie, lorsque le gars voulut recommencer, Simon lui jeta ces mots à la tête, comme il aurait fait d'une pierre : "Il s'appelle Philippe, mon papa. » (Id., Contes et nouvelles, I, Gallimard [Pléiade], I974-I979: 79)

Le processus de généralisation a continué jusqu'à l'heure actuelle, où l'écrasante majorité des auteurs ne pratiquent plus que la construction indirecte. Or, ce processus n'est jamais allé jusqu'à éliminer tout à fait l'ancienne construction directe. Contrairement à ce qu'on dit parfois (cf. Fournier \& Fuchs 1999), il y a eu tout au long du XX $\mathrm{XX}^{\mathrm{e}}$ siècle des auteurs qui, cultivant l'archaïsme, n'ont pas adopté la nouvelle syntaxe et qui, pour des raisons contextuelles surtout, faisaient alterner les deux constructions, par exemple Proust, Gide, Mauriac, Duhamel (cf. Eriksson 2006 : 922). Plus près de nous, Michel Tournier (43-44), François Nourissier $(45-46)$ et Jacques Borel $(47-48)$ représentent cette attitude :

(43) [...] elle prétendait vaquer à ses occupations intérieures en le portant agrippé à son flanc, comme font les guenons leur petit (Tournier, Le Coq de bruyère, Gallimard, 1978 : 100)

(44) En somme, je fécondais cette terre comme j'aurais fait une épouse (Id., Vendredi ou les Limbes du Pacifique, Folio, 1975 : 229)

(45) La terreur habite la maison comme fait l'eau l'étang (Nourissier, Le Maître de maison, Grasset, I968 : I2)

(46) Elle le traitait de plus en plus souvent comme les bien-portants font les déprimés, les mélancoliques (Id., L’Empire des nuages, Grasset, I98 I : I05)

(47) Ma grand-mère [...] a secoué longuement son parapluie, un peu comme on fait la salade dans son panier (Borel, Le Retour, Gallimard, I970: I56) 
(48) J'acceptai, bien que je me fusse toujours senti assez peu concerné par cette œuvre, étranger à ses problèmes, et qu'elle ne m'eût jamais marqué, comme elle faisait les gens de mon temps (Id., L'Adoration, I965:599)

Pour la question de la chronologie des quatre prépositions qui entrent en concurrence, il semble que de soit la plus ancienne. C'est d'elle, on l'a vu, que se sert Rabelais. L'unique exemple cité par Moignet (I960 : I I8) pour montrer l'emploi de la préposition $a ̀$ en ancien français n'est pas très probant, parce que le verbe de la principale y est à la voix passive et que, par conséquent, il n'y a pas, à strictement parler, d' " objet direct " (49) (pour un autre exemple, plus probant, datant de la fin du XVe siècle, voir Ponchon I994 : 26I). Pour notre part, nous en avons relevé quelques exemples dans l'Histoire comique de Francion de Charles Sorel, publié en I 623 (50). Elle se rencontre encore, bien que rarement, au XX $X^{\mathrm{e}}$ siècle (5I) :

(49) Et quant ele fu trespassee, ele fu enterree, si hautement comme l'an doit fere à si haute dame (Mort Artu, Appendice)

(50) J'ay esté plusieurs fois voir des Courtisannes de cette ville que j'ay escroquées par plaisir, ainsi que j'avois accoustumé de faire à celle de France (Sorel, Histoire comique de Francion, Gallimard [Pléiade], I958: 52I)

(5I) Leur maître, un jeune gentilhomme courteaud et rougeaud, ne cessait de les encourager de la voix et du geste, comme on fait aux chiens (Anatole France, La Rôtisserie de la Reine Pédauque, Calmann-Lévy, I959: I2I)

Quant à la préposition pour, elle semble remonter au début du XVIII ${ }^{\mathrm{e}}$ siècle. Nous en avons relevé quelques exemples dans les Mémoires de Saint-Simon (52). C'est pourtant le XX $\mathrm{XX}^{\mathrm{e}}$ siècle qui en consacre l'usage et qui la voit atteindre une fréquence relativement élevée (53-54):

(52) La vérité est que les Electeurs évitèrent de le voir, comme ils firent pour M. de Chevreuse (Saint-Simon, Mémoires, Gallimard [Pléiade], II, I948-I955:650)

(53) - Vous auriez tenté de l'empoisonner comme vous l'auriez fait, paraitil, pour votre première femme (Jean Hougron, La Chambre, Hachette, I982:267)

(54) C'est à peine si on le tenait à l'écart, comme on le fait d'ordinaire pour les bourreaux qui en prennent une importance quasi tragique (Françoise Mallet-Joris, Trois âges de la nuit, Grasset, I968 : 3 I9) 
Avec, enfin, est la nouvelle venue des quatre prépositions. Nous en avons relevé un exemple isolé chez Rimbaud (55). Or, cet exemple mis à part, il faut attendre les années quarante pour la voir s'installer dans l'usage de façon permanente (56). Elle est en voie d'expansion (57-58), en conformité, peut-être, avec le développement général qu'on observe à l'heure actuelle dans l'usage prépositionnel du français, qui tend à réserver à avec le rôle d'une préposition à tout faire :

(55) Il me jeta un bonjour sec, fronça le nez en jetant un coup d'œil sur mes souliers à cordons noirs, et s'en alla devant moi, les mains dans ses deux poches, ramenant en devant sa robe de chambre, comme fait l'abbé *** avec sa soutane, et modulant ainsi à mes regards sa partie inférieure (Rimbaud, CEuvres diverses, Un coeur sous une soutane, Gallimard [Pléiade], I962:200)

(56) Un homme volumineux s'approcha de Leila, la prit par la taille et la souleva, très haut, comme on le fait avec les tout-petits (Elsa Triolet, Le Monument, Folio, I976:40)

(57) Il a d'abord poignardé l'amant [...] cependant que la femme hurlait, tentait de fuir. Puis il l'a égorgée, elle, comme on le fait avec les moutons (Michel del Castillo, La Gloire de Dina, Seuil, I988:97)

(58) Ils ne raffinent pas, ils exécutent sommairement d'une balle ou d'un coup de baïonnette, rapidement, mais selon un certain ordre, comme on fait avec les animaux à l'abattoir (Lucien Bodard, La Vallée des roses, Le Livre de Poche, I982 : 32I)

Cette expansion concerne en premier lieu la langue non-littéraire. Ainsi, sur Google, on trouve actuellement un nouveau développement très rapide : la fréquence de la préposition avec y dépasse de loin celle de de. C'est, nous semble-t-il, par un processus de grammaticalisation qu'elle y est arrivée ( $c f$. Eriksson 2008). L'affaiblissement du lien transitif qu'elle opère entre proverbe et objet - plus important que celui des autres prépositions - a pour effet d'augmenter la capacité " représentative » du proverbe et de lui faire étendre, ainsi, la portée de sa représentation à des compléments adverbiaux du genre de ceux (en italique) que contient la comparative dans les exemples (59-6I); de préposition à sens plein avec est devenue instrument syntaxique :

(59) Chez Ndiaye on avait même installé l'ordinateur dehors sur une chaise, comme on le fait avec la télé

(62.2 I0.2I 8.I74/ xof/spip/article.php3 ?id_article=40) 
(60) Les nazis ont déshumanisé les juifs, en les identifiant par un numéro tatoué sur le bras, comme on le fait avec les animaux (www.sixmillion.org/Cadres/textfrancais.html)

(6I) On le suit par peur et par obligation, et non par admiration et par amour comme on le fait avec le leader (pages.infinit.net/espoir3/le_dominateur_et_le_domine.htm)

De là, on en est venu à une situation où l'usage d'avec tend à se généraliser, comme en témoigne le recours à avec même en l'absence de compléments adverbiaux, avec une représentation se réduisant au seul verbe principal (en italique) :

(62) En agissant ainsi, il vous respectera comme on le fait avec un vrai partenaire (www.jeanpierrelauzier.com/client.html)

(63) Tous commencent à l'appeler Nicole, comme on le fait avec une personne qui fait partie de la famille (www.socialist-utopia.org/node/view/3 I 5)

Preuve particulièrement concluante de l'existence d'un tel processus de grammaticalisation, la préposition tend à rester avec même quand, dans le cas d'un objet prépositionnel, le verbe de la principale prend une préposition autre qu'avec (64-65) (d'autres exemples dans Eriksson 2008). Le même phénomène s'observe en ce qui concerne l'objet indirect (datif) (66-67). Chose remarquable, on constate qu'en anglais et en suédois, le même processus de grammaticalisation est en train de conférer aux prépositions with (68-69) et med (70-7I) le rôle d'outils syntaxiques :

(64) [...] j'ai commencé à fumer plus d'un paquet par jour, en me délectant de chaque cigarette comme on le fait avec du chocolat en ouvrant la tablette (www.hi3.fr/dotclear/index.php?2006/o6/I8/98-tenue-d-eve)

(65) Toujours laisser faire car c'est normal qu'elle veuille redevenir bébé, elle aimerait bien qu'on s'occupe d'elle comme on le fait avec sa petite sœur

(www.infobebes.com/htm/bebe/sos-grande-soeur-jalouse,m-I 8 I 865 . aspx)

(66) On ne dit pas "non » $\grave{a}$ un perroquet comme on le fait avec un chien (www.csp-valleedesforts.com/fumepas.htm) 
(67) J'aurais dû lui donner les questions d'avance comme on le fait avec certains VIP

(www.gapali.be/gapali/20030913/25ans.html)

(68) Try looking at things from a new point of view just as you do with your writing

(www.cameraontheroad.com/?p=244)

(69) You have to work with your feelings first and make yourself feel as much for your disfavored child as you do with the favored one (www.webmd.com/content/article/I/I700_5068I)

(70) Man måste våga prata om det, precis som man gör med nära-dödenupplevelser (paranormal.se/topic/kundaliniskildring_elisabeth.html)

(7I) Man ska förhålla sig till drickandet som man gör med andningen, sömnen och blinkandet - låt kroppen sköta det (strangnet.se/blog/index.php/sv?cat=79)

\section{Remarque finale}

L'évolution historique en français de la construction «objective » en comparative se caractérise par l'introduction d'une préposition entre proverbe et objet, puis par la généralisation de cet emploi, si bien que, de nos jours, on ne trouve l'ancienne construction directe, en littérature française, que chez une poignée d'auteurs qui se piquent de ne pas reculer devant les archaïsmes. Il s'agit d'une évolution lente, quoique ininterrompue et irréversible.

Le motif de l'insertion de la préposition semble résider dans le relâchement que celle-ci opère dans la transivité du proverbe, relâchement causé par le besoin qu'on a éprouvé de faire étendre la portée de la représentation de faire à l'ensemble du syntagme verbal de la principale. Par là, la préposition est devenue un outil grammatical, plus ou moins vidé de son contenu lexical, comme c'est le cas du proverbe lui-même. La comparative offre donc ici la scène d'une rencontre de deux éléments définis grammaticalement. En effet, c'est, selon nous, à partir de l'incompatibilité d'un élément à base lexicale avec un élément à base grammaticale qu'il faut expliquer le maintien de la préposition la plus récente, avec - plus ouverte à la grammaticalisation que ses concurrents prépositionnels - même quand le verbe de la principale se construit avec une autre préposition. La constatation du même phénomène en anglais et en suédois contribue encore, nous semble-t-il, à montrer le bien-fondé de cette conclusion. 


\section{Références}

Apothéloz, Denis. 1995. Rôle et fonctionnement de l'anaphore dans la dynamique textuelle. Genève : Droz.

Brunot, Ferdinand. I966 [1905]. Histoire de la langue française des origines à nos jours. Vol. I : De l'époque latine à la Renaissance. Paris: Armand Colin.

Damourette, Jacques \& Edouard Pichon. 1936. Des mots à la pensée. Essai de grammaire de la langue française. Vol. V. Paris : D'Artrey.

Eriksson, Olof. I985. La suppléance verbale en français moderne. Göteborg : Acta Universitatis Gothoburgensis (Romanica Gothoburgensia XXV).

Eriksson, Olof. 2006. «Un cas de suppléance verbale en français comme illustration d'une méthode d'analyse linguistique ». Revue Belge de Philologie et d'Histoire, 84. 913-928. Publié aussi in : Lenoble-Pinson, Michèle \& Christian Delcourt (dir.), Le point sur la langue française. Hommage à André Goosse. Bruxelles : Le Livre Timperman, 2006. 379-394.

Eriksson, Olof. 2008. " A contrastive study of proverbalization ». Languages in Contrast, 8. 235-26I.

Eriksson, Olof. 20I0. "Den så kallade med-frasen i kontrastiv belysning ". Språk och stil, 20. 50-8I.

Fournier, Nathalie \& Catherine Fuchs. I999. "L'évolution du statut de faire dans les comparatives en comme et la constitution du groupe verbal (XVII ${ }^{e_{-}}$ $\mathrm{XX}^{\mathrm{e}}$ siècles) ». Verbum, $2 \mathrm{I}:$ 3. 289-322.

Halliday, Michael \& Ruqaiya Hasan. 1976. Cohesion in English. London : Longman.

Hjelmslev, Louis. I937. "La nature du pronom ». (Sans dir.), Mélanges de linguistique et de philologie offerts à Jacq. van Ginneken. Paris : Klincksieck. $5 \mathrm{I}-58$.

Le Bidois, Georges \& Robert Le Bidois. I97I [1938]. Syntaxe du français moderne. Vol. II. Paris : Picard.

Livet, C.-L. I 896. Lexique de la langue de Molière. Vol. II. Paris : H. Welter.

Marty-Laveaux, C. 1868. Lexique de la langue de P. Corneille. Paris : Hachette.

Moignet, Gérard. 1960. "La suppléance du verbe en français ». Le français moderne, $28:$ I. I3-24 et $28: 2$. IO7-I 24.

Noreen, Adolf. I904-I9I2. Vårt språk. Nysvensk grammatik i utförlig framställning. Vol V. Lund : Gleerup.

Pinchon, Jacqueline. 1972. Les pronoms adverbiaux en et y. Problèmes généraux de la représentation pronominale. Genève : Droz. 
Ponchon, Thierry. 1994. Le verbe faire en français médiéval. Genève : Droz.

Quillacq, J.-A. I903. La langue et la syntaxe de Bossuet. Tours : Alfred Cattier.

Regnier, Henri de, I892. Lexique de la langue de J. de La Fontaine. Vol. I. Paris : Hachette.

Teleman, Ulf, Staffan Hellberg \& Erik Andersson. I999a. Svenska Akademiens grammatik. Vol. I : Inledning. Register. Stockholm : Norstedts Ordbok.

Teleman, Ulf, Staffan Hellberg \& Erik Andersson. I999b. Svenska Akademiens grammatik. Vol. II : Ord. Stockholm : Norstedts Ordbok.

Thorell, Olof. I977 [1973]. Svensk grammatik. Stockholm : Esselte. 